\title{
A particle-number-conserving Bogoliubov method which demonstrates the validity of the time-dependent Gross-Pitaevskii equation for a highly condensed Bose gas
}

Final version 27 February 1997

\author{
C. W. Gardiner \\ Physics Department, Victoria University of Wellington, Wellington, New Zealand
}

The Bogoliubov method for the excitation spectrum of a Bose-condensed gas is generalized to apply to a gas with an exact large number $N$ of particles. This generalization yields a description of the Schrödinger picture field operators as the product of an annihilation operator $A$ for the total number of particles and the sum of a "condensate wavefunction" $\xi(\mathbf{x})$ and a phonon field operator $\chi(\mathbf{x})$ in the form $\psi(\mathbf{x}) \approx A\{\xi(\mathbf{x})+\chi(\mathbf{x}) / \sqrt{N}\}$ when the field operator acts on the $N$ particle subspace. It is then possible to expand the Hamiltonian in decreasing powers of $\sqrt{N}$, an thus obtain solutions for eigenvalues and eigenstates as an asymptotic expansion of the same kind. It is also possible to compute all matrix elements of field operators between states of different $N$.

The excitation spectrum can be obtained by essentially the same method as Bogoliubov only if $\xi(\mathbf{x})$ is a solution of the time independent Gross-Pitaevskii equation for $N$ particles and any chemical potential $\mu$ which yields a valid and stable solution of the Gross-Pitaevskii equation. The treatment within a subspace of fixed $N$ is identical in form to that usually used, but the interpretation of the operators is slightly different.

A time-dependent generalization is then made, yielding an asymptotic expansion in decreasing powers of $\sqrt{N}$ for the equations of motion. In this expansion the condensate wavefunction has the time-dependent form $\xi(\mathbf{x}, t)$, and the condition for the validity of the expansion is that $\xi(\mathbf{x}, t)$ satisfies the time-dependent Gross-Pitaevskii equation $\partial \xi / \partial t=$ $-\left(\hbar^{2} / 2 m\right) \nabla^{2} \xi+V \xi+N u|\xi|^{2} \xi$.

The physics is then described in a kind of interaction picture, called the condensate picture, in which the phonon operator can be expressed as $\chi(\mathbf{x}, t)=\sum_{k} \xi_{k}(\mathbf{x}, t) \alpha_{k}$, where the operators $\alpha_{k}$ are time independent annihilation operators, and the state-vector has a time evolution described by a Schrödinger equation in which the Hamiltonian is a timedependent quadratic form in the phonon creation and annihilation operators, whose coefficients are explicitly determined in terms of the time-dependent condensate wavefunction $\xi(\mathbf{x}, t)$

\section{INTRODUCTION}

Two central tools in the description of a Bosecondensed gas are the Bogoliubov method $11-1$ and the Gross-Pitaevskii equation [5] in both its time indepen- dent and its time dependent guises. Because Bose condensates [6] can now be be experimentally produced, there has been renewed interest in both of these tools (9.8.10 13. 14 24.

As it is presently formulated, Bogoliubov's method treats the condensate operators a c-numbers. One consequence of this is that the resulting approximate Hamiltonian does not conserve the total number of particles. The enforcement of number conservation in the mean only leads to a description which is essentially confined to a subspace with a single value of the mean number of particles.

Another difficulty is that, although there are derivations of the validity of the time independent GrossPitaevskii equation as a description of the condensate ground state wavefunction (these appear largely as consequences of the adaptation of the Bogoliubov method to a trapped condensate), there does not appear to be any derivation of the validity of the time-dependent GrossPitaevskii equation as a description of the motion of a trapped condensate.

This paper will solve both of these problems and show that their solutions are strongly connected.

We will firstly show how to modify Bogoliubov's argument in such a way as not to break the conservation of particle number. This modification yields a description of the particle field operator $\psi(\mathbf{x})$ in the form

$$
\psi(\mathbf{x}) \approx A\left(\xi(\mathbf{x})+\frac{1}{\sqrt{N}} \chi(\mathbf{x})\right)
$$

where $A$ is an annihilation operator such that the eigenvalue of $A^{\dagger} A$ is $N$, the total number of particles, $\xi(\mathbf{x})$ is the condensate wavefunction, and $\chi(\mathbf{x})$ is a phonon field operator. The Bogoliubov Hamiltonian is expressed in terms of these phonon operators, and the nonconservation of phonons which arises is not unexpected. The eigenstates of this Bogoliubov Hamiltonian are nevertheless all states with exactly $N$ particles, so there is no doubt that particle number conservation is not violated. The method is approximate, of course, but the accuracy of the approximations made is exactly the same as that of the usual Bogoliubov method.

It is clear that the separation of the phonon concept from the particle concept that enables the method to succeed. To emphasize the validity of the the phonon concept we show that our expression for $\psi(\mathbf{x})$ can be used to demonstrate that the quantized phase of $\psi(\mathbf{x})$ is the velocity potential operator, and that the operator of density 
fluctuations is its canonical conjugate, as is well known 3.

The treatment of the spatially inhomogeneous case requires more care in defining exactly what the expansion procedure is. The Bogoliubov method and the GrossPitaevskii equations are both used to describe the weakly interacting Bose gas; the requirement that the gas is weakly interacting is formalized in our treatment by requiring the interaction potential $u$ to be of order of magnitude $1 / N$; that is, we write $u=\tilde{u} / N$, and carry out the asymptotic expansion in decreasing powers of $\sqrt{N}$ at fixed $\tilde{u}$.

The treatment is then similar for the case of the time independent or the time dependent condensates. The formula (11) is substituted in the Hamiltonian, and the terms of different degree in $\sqrt{N}$ grouped together yielding

$$
H=N \mathcal{H}_{1}+\sqrt{N} \mathcal{H}_{2}+\mathcal{H}_{3}
$$

$\mathcal{H}_{1}$ is found to be a c-number, $\mathcal{H}_{2}$ is linear in phonon operators and $\mathcal{H}_{3}$ is quadratic in phonon operators.

In the time-independent case the ground state is found by minimizing $\mathcal{H}_{1}$, and this means that $\xi(\mathbf{x}, t)$ must satisfy the time-independent Gross-Pitaevskii equationthis also means that the linear term $\mathcal{H}_{2}$ vanishes, and the excitation spectrum is given by diagonalizing the quadratic part $\mathcal{H}_{3}$.

The description of the inhomogeneous condensate that results is almost isomorphic to that originally used by Fetter 7. when restricted to a subspace of definite $N$, and thus is in agreement with other recent calculations. However, we obtain in addition a description of the relationship between the states for different $N$, and in particular are able to present the matrix elements of the field operators between states of $N$ and $N+1$ particles. (It was the need to have these in a description of condensate growth which motivated this work originally.)

In the time-dependent case the logic is slightly different. In general there is an explicit time-dependence proportional to $\sqrt{N}$ in $\chi(\mathbf{x}, t)$ as a result of the representation of the time-independent $\psi(\mathbf{x})$ operator in terms of a time dependent condensate wavefunction $\xi(\mathbf{x}, t)$, and this being of order of magnitude $\sqrt{N}$ contradicts the asymptotic representation in the form (11). The part proportional to $\sqrt{N}$ however can be made to cancel with the implicit time dependence arising for $\sqrt{N} \mathcal{H}_{2}$ provided $\xi(\mathbf{x}, t)$ satisfies the time dependent Gross-Pitaevskii equation: thus it is only possible to get an asymptotic expansion of the form (1) if $\xi(\mathbf{x}, t)$ is a solution of the time dependent Gross-Pitaevskii equation. The remaining time dependence can adjusted by a suitable choice phonon mode functions $\xi_{k}(t)$ to yield a representation in terms of time independent phonon annihilation operators, and by going into an appropriate interaction picture - the condensate picture - the time dependence of the state vector is given by a Schrödinger equation $\mathcal{H}_{3}(t)|\Phi, t\rangle=i \hbar d|\Phi, t\rangle / d t$. Since the number of atoms is fixed, this gives a full description of the system; the condensate wavefunction sat- isfies the time-dependent Gross-Pitaevskii equation, and the motion of the residual quantized phonons is given by $\mathcal{H}_{3}(t)$

\section{A NUMBER CONSERVING BOGOLIUBOV TRANSFORMATION FOR THE HOMOGENEOUS BOSE GAS}

Let us start with the problem of the weakly interacting Bose gas confined in a large box, with no explicit trapping potential. The Hamiltonian in momentum space is written

$$
\begin{aligned}
H= & \sum_{\mathbf{k}} \hbar \omega_{\mathbf{k}} a_{\mathbf{k}}^{\dagger} a_{\mathbf{k}} \\
& +\frac{u}{2} \sum_{\mathbf{k}_{1}, \mathbf{k}_{2}, \mathbf{k}_{3}, \mathbf{k}_{4}} a_{\mathbf{k}_{1}}^{\dagger} a_{\mathbf{k}_{2}}^{\dagger} a_{\mathbf{k}_{3}} a_{\mathbf{k}_{4}} \delta_{\mathbf{k}_{1}+\mathbf{k}_{2}, \mathbf{k}_{3}+\mathbf{k}_{4}} .
\end{aligned}
$$

The basis states are usually written

$$
\left|n_{0}, \mathbf{n}\right\rangle
$$

where $\mathbf{n} \equiv\left\{n_{\mathbf{k}}\right\}$ represents the vector of occupation numbers of all particles with non-zero momentum, and the dependence on $n_{0}$ is explicitly separated. Now let us write them in a form which eliminates reference to $n_{0}$ by using the total number $N=n_{0}+\sum_{\mathbf{k}} n_{\mathbf{k}}$, in the form

$$
|N, \mathbf{n}\rangle
$$

We define the operators $A, \mathcal{N}$ and $\alpha_{\mathbf{k}}$ as follows

$$
\begin{aligned}
A|N, \mathbf{n}\rangle & =\sqrt{N}|N-1, \mathbf{n}\rangle \\
\mathcal{N} & \equiv A^{\dagger} A \\
\alpha_{\mathbf{k}} & =\frac{1}{\sqrt{\mathcal{N}}} a_{0}^{\dagger} a_{\mathbf{k}}
\end{aligned}
$$

The operators $\alpha_{\mathbf{k}}$ are essentially phonon operators, while $A$ and $\mathcal{N}$ are operators which refer to the total numbers of particles. Since the phonon operators $\alpha_{\mathbf{k}}$ commute with the total number operator $\mathcal{N}$, we can make approximations involving the phonon operators without violating the conservation of total numbers of particles.

\section{Relationship between $a_{0}$ and $A$}

The operator $A$ reduces the total number of particles by 1 , without changing the number of $\mathbf{k} \neq 0$ particles; it is thus proportional to the operator $a_{0}$. In fact the precise relationship is

$$
\begin{aligned}
a_{0}|N, \mathbf{n}\rangle & =\sqrt{n_{0}}|N-1, \mathbf{n}\rangle \\
& =\sqrt{1-\frac{\sum_{\mathbf{k} \neq 0} n_{\mathbf{k}}}{N}} A|N, \mathbf{n}\rangle
\end{aligned}
$$


If $N$ is very large compared with $\sum_{\mathbf{k} \neq 0} n_{\mathbf{k}}$-that is, the system is Bose condensed - it is clear that we may make the approximation

$$
\begin{aligned}
a_{0}|N, \mathbf{n}\rangle & =A\left(1-\frac{\sum_{\mathbf{k} \neq 0} n_{\mathbf{k}}}{2 N}\right)|N, \mathbf{n}\rangle \\
& \approx A|N, \mathbf{n}\rangle .
\end{aligned}
$$

In what follows we will be expanding in inverse powers of $N^{1 / 2}$, and it will sometimes be necessary to keep the more accurate form (11).

\section{Relationship between $a_{\mathbf{k}}$ and $\alpha_{\mathbf{k}}$}

One can similarly show that one can write

$$
\begin{aligned}
a_{\mathbf{k}}|N, \mathbf{n}\rangle & \approx \frac{A \alpha_{\mathbf{k}}}{\sqrt{N+1-\sum_{\mathbf{k}} n_{\mathbf{k}}}}|N, \mathbf{n}\rangle \\
& \approx \frac{1}{\sqrt{N}}\left(1+\frac{\sum_{\mathbf{k} \neq 0} n_{\mathbf{k}}-1}{2 N}\right) A \alpha_{\mathbf{k}}|N, \mathbf{n}\rangle \\
& \approx A \frac{\alpha_{\mathbf{k}}}{\sqrt{N}}|N, \mathbf{n}\rangle
\end{aligned}
$$

however we will not find it necessary to use anything other than the simple form (15).

\section{Commutators of the $\alpha_{\mathbf{k}}$}

When acting on such condensed states with large $N$, we also find that we can approximate the commutator

$$
\begin{aligned}
{\left[\alpha_{\mathbf{k}}, \alpha_{\mathbf{k}^{\prime}}^{\dagger}\right] } & =\delta_{\mathbf{k k}^{\prime}}-\frac{1}{N} a_{\mathbf{k}} a_{\mathbf{k}^{\prime}}^{\dagger} \\
& \approx \delta_{\mathbf{k k}^{\prime}} .
\end{aligned}
$$

\section{Validity of the approximations}

The approximations made are accurate to the same order in $N$ as those usually assumed in the Bogoliubov theory. For clarity we have written equations such as (6, 9. 13 etc., as explicitly acting on a state with $N$ particles. However in the remainder of the paper we will simply write equalities between operators, which are understood to be valid when these operators act on a vector in the subspace of $N$ particles.

\section{A. Transformation of the Hamiltonian}

The Hamiltonian (3) is now approximated firstly, as is usual in Bogoliubov theory by dropping the terms in the interaction which do not involve at least two operators $a_{0}, a_{0}^{\dagger}$, and then secondly by making the replacements (and appropriate Hermitian conjugates)

$$
\begin{aligned}
a_{0}^{\dagger} a_{\mathbf{k}} & \rightarrow \alpha_{\mathbf{k}} \sqrt{\mathcal{N}} \\
a_{0}^{\dagger} a_{0} & \rightarrow \mathcal{N} \\
a_{\mathbf{k}}^{\dagger} a_{\mathbf{k}} & \rightarrow \alpha_{\mathbf{k}}^{\dagger} \alpha_{\mathbf{k}}
\end{aligned}
$$

in the Hamiltonian. Notice that for all of these replacements the left hand sides have essentially the same action on a number state as the right hand sides, but with a modified coefficient; that is for all of them the non-zero matrix elements occur for the same states, and the coefficients are almost equal, in contrast to the usual Bogoliubov transformation, which changes the essential nature of the operators in the number state basis.

As in the usual Bogoliubov method, the term involving $a_{0}^{\dagger} a_{0}^{\dagger} a_{0} a_{0}$ has to be treated more accurately, effectively by using the more accurate approximation (11), giving

$$
\begin{aligned}
a_{0}^{\dagger} a_{0}^{\dagger} a_{0} a_{0} & =a_{0}^{\dagger} a_{0} a_{0}^{\dagger} a_{0}-a_{0}^{\dagger} a_{0} \\
& \approx \mathcal{N}^{2}-\mathcal{N}-2 \mathcal{N} \sum_{\mathbf{k}} a_{\mathbf{k}}^{\dagger} a_{\mathbf{k}} \\
& \approx \mathcal{N}^{2}-\mathcal{N}-2 \mathcal{N} \sum_{\mathbf{k}} \alpha_{\mathbf{k}}^{\dagger} \alpha_{\mathbf{k}}
\end{aligned}
$$

Although in this case it is usual to set $\omega_{0}=0$, for consistency with the inhomogeneous case we do not make this assumption, and this requires a similar correction to the term $\hbar \omega_{0} a_{0}^{\dagger} a_{0}$. The Hamiltonian (3) then becomes

$$
\begin{aligned}
H= & \frac{1}{2} u\left(\mathcal{N}^{2}-\mathcal{N}\right)+\sum_{\mathbf{k} \neq 0} \hbar\left(\omega_{\mathbf{k}}-\omega_{0}\right) \alpha_{\mathbf{k}}^{\dagger} \alpha_{\mathbf{k}} \\
& +\frac{u \mathcal{N}}{2} \sum_{\mathbf{k} \neq 0}\left\{2 \alpha_{\mathbf{k}}^{\dagger} \alpha_{\mathbf{k}}+\alpha_{\mathbf{k}} \alpha_{-\mathbf{k}}+\alpha_{\mathbf{k}}^{\dagger} \alpha_{-\mathbf{k}}^{\dagger}\right\}
\end{aligned}
$$

It is to be understood that this operator form is valid on all states, including superpositions of states with different numbers of particles, as long as only states with large eigenvalues of $\mathcal{N}$ are included. The Hamiltonian can be diagonalized for any eigenvalue of $\mathcal{N}$, to get the usual Bogoliubov spectrum; the eigenstates are simultaneous eigenstates of $\mathcal{N}$ and $H$.

\section{Asymptotic expansion}

The expression of the Hamiltonian in the form (22) can be put in the form of an asymptotic expansion in inverse powers of $N$ in the sense that the restriction of $H$ to a subspace of definite $N$ is approximated by (22) for sufficiently large $N$, provided it is understood that $u$ is itself a small quantity of order of magnitude $1 / N$. Under that condition it can be checked that approximations made in its derivation affect only the terms of lower order in $N$; i.e., the corrections are of order $1 / N$. The requirement that $u$ be "small" amounts to an assumption that 
the kinetic plus trapping potential terms, and interaction energy terms in (22), are of the same order of magnitude. A formal statement of this requirement can be made by writing $u=\tilde{u} / N$ in (22), and then solving the problem by making an expansion in appropriate decreasing powers of $N$.

The Hamiltonian in this form has two major advantages over the deceptively similar conventional form. The most significant is that the conservation of total numbers of particles is maintained; the quasiparticles on the other hand appear only as phonons, and are not conserved. The second major advantage is that the approximation method is a systematic expansion in inverse powers of the large quantity $N$, which has a definite value in any case one considers.

\section{Expression of field operators in terms of the velocity potential operator}

The consequences of this method on the representation of the field operators are interesting. We can show that the representation of phase and density fluctuations as in [3] is a natural consequence.

The field operator is

$$
\begin{aligned}
\psi(\mathbf{x}) & =\frac{1}{\sqrt{V}}\left(a_{0}+\sum_{\mathbf{k} \neq 0} a_{\mathbf{k}} e^{i \mathbf{k} \cdot \mathbf{x}}\right) \\
& \approx \frac{A}{\sqrt{V}}\left(1+\frac{1}{\sqrt{N}} \sum_{\mathbf{k} \neq 0} \alpha_{\mathbf{k}} e^{i \mathbf{k} \cdot \mathbf{x}}\right)
\end{aligned}
$$

We now introduce the Bogoliubov transformation, approximated by assuming $\mathbf{k}$ is very small (though this is not an essential assumption, and is introduced only to make it match up with [3]), as

$$
\alpha_{\mathbf{k}}=\frac{b_{\mathbf{k}}-b_{-\mathbf{k}}^{\dagger}}{\sqrt{2 \hbar k / m v(N)}}+\sqrt{\frac{\hbar k}{2 m v(N)}} b_{-\mathbf{k}}^{\dagger}
$$

where $v(N)=\sqrt{u N / m}$ is the speed of sound for long wavelengths. Clearly, the second term is much smaller than the first for small $\mathbf{k}$, so we keep only the first part initially. We then find that

$$
\begin{aligned}
\psi(\mathbf{x}) & \approx \frac{A}{\sqrt{V}}\left[1+\sum_{\mathbf{k} \neq 0} \sqrt{\frac{m v}{2 \hbar k N}}\left(b_{\mathbf{k}} e^{i \mathbf{k} \cdot \mathbf{x}}-b_{\mathbf{k}}^{\dagger} e^{-i \mathbf{k} \cdot \mathbf{x}}\right)\right] \\
& \approx \frac{A}{\sqrt{V}} \exp (i \Phi(\mathbf{x}))
\end{aligned}
$$

since

$$
\Phi(\mathbf{x})=-i \sum_{\mathbf{k} \neq 0} \sqrt{\frac{m v}{2 \hbar k N}}\left\{b_{\mathbf{k}} e^{i \mathbf{k} \cdot \mathbf{x}}-b_{\mathbf{k}}^{\dagger} e^{-i \mathbf{k} \cdot \mathbf{x}}\right\}
$$

is small.

This matches up exactly with (27.1) of 3]. That is, the operator $\Phi$ is the quantized velocity potential operator, with the substitution $b_{\mathbf{k}} \rightarrow i c_{\mathbf{k}}$ to match up with their notation. If we now include the next order term we find density fluctuations as well. Using the full form (25) for the density

$$
\begin{aligned}
\psi^{\dagger}(\mathbf{x}) \psi(\mathbf{x}) & \approx \frac{N}{V}+\frac{1}{V} \sum_{\mathbf{k} \neq 0} \sqrt{\frac{N \hbar k}{2 m v}}\left\{b_{\mathbf{k}} e^{i \mathbf{k} \cdot \mathbf{x}}+b_{\mathbf{k}}^{\dagger} e^{-i \mathbf{k} \cdot \mathbf{x}}\right\} \\
& =\rho_{0}+\delta \rho(\mathbf{x})
\end{aligned}
$$

giving the correct operator for the density fluctuations, as in [3] (24.10).

In this limit that only long wavelengths are involved, the Hamiltonian (22) can be rewritten in terms of a part related to sound waves, as in [3], and an additional purely $\mathcal{N}$ dependent part:

$$
\begin{aligned}
H= & \frac{1}{2} u\left(\mathcal{N}^{2}-\mathcal{N}\right)+E_{0}(\mathcal{N}) \\
& +\frac{1}{2}: \int d^{3} \mathbf{x}\left\{\rho_{0}(\nabla \cdot \Phi(\mathbf{x}))^{2}+v(\mathcal{N})^{2} \frac{\delta \rho(\mathbf{x})^{2}}{\rho_{0}}\right\}: .
\end{aligned}
$$

Here $E_{0}(\mathcal{N})$ is the Bogoliubov ground state energy.

Notice that not only do $\rho_{0}$ and $v(N)$ depend on $N$, but that the very definitions of the $\Phi(\mathbf{x})$ and $\delta \rho(\mathbf{x})$ also depend on $N$. However, if we believe that a superselection rule applies whereby only eigenstates of $N$ occur in nature, the correct treatment of states with uncertain total numbers of particles must arise from an incoherent superposition of solutions, for various $N$, of the equations of motion arising from (31).

\section{THE SPATIALLY INHOMOGENEOUS CASE}

The spatially inhomogeneous situation, which can arise either by the existence of vortices or because there is a trapping potential confining the gas, was first treated by Fetter [7], and in the case of a trapped gas, has been treated more recently in 8,11, 13.

The standard formalism, as used by Fetter [7], is based on the work of Hugenholtz and Pines [26] which in summary, shows that the correct ground state and excitation spectrum is given by the following prescription:

1. Replace $a_{0}^{\dagger} \rightarrow \sqrt{N}, a_{0} \rightarrow \sqrt{N}$ in both $H$ and $\mathcal{N}$.

2. For a given $\mu$, find the value of $N$ and the state which minimize $\langle K\rangle$, where $K=H-\mu N$. This gives the ground state energy.

3. The excited states are given by the higher eigenstates of $K$ with $a_{0}^{\dagger} \rightarrow \sqrt{N}, a_{0} \rightarrow \sqrt{N}$. By neglecting terms in $N$ of order less than $N$, one obtains 
the Bogoliubov Hamiltonian, which can be exactly diagonalized, but Hugenholtz and Pines in fact included more terms than these in their evaluation of the ground state energy, and hence obtain a more accurate result.

It is particularly important to note that there is no basis for using $K$ without setting $a_{0}^{\dagger} \rightarrow \sqrt{N}, a_{0} \rightarrow \sqrt{N}$, as was done by [11, which can be seen as an initial attempt to get a Bogoliubov description which covers a range of $N$ values.

\section{A. Formulation of the modified Bogoliubov method}

\section{Expression of the field operators in terms of phonon operators}

Our treatment will be based on that already used for the spatially homogeneous case. We therefore consider the general case for which the Hamiltonian is

$$
\begin{aligned}
H= & -\frac{\hbar^{2}}{2 m} \int d^{3} \mathbf{x} \psi^{\dagger}(\mathbf{x}) \nabla^{2} \psi(\mathbf{x})+\int d^{3} \mathbf{x} \psi^{\dagger}(\mathbf{x}) V(\mathbf{x}) \psi(\mathbf{x}) \\
& +\frac{u}{2} \int d^{3} \mathbf{x} \psi^{\dagger}(\mathbf{x}) \psi^{\dagger}(\mathbf{x}) \psi(\mathbf{x}) \psi(\mathbf{x})
\end{aligned}
$$

We will make the substitution

$$
\begin{aligned}
\psi(\mathbf{x}) & =\left(a_{0} \xi(\mathbf{x})+\sum_{k} \xi_{k}(\mathbf{x}) a_{k}\right) \\
& \approx A\left(\xi(\mathbf{x})+\frac{1}{\sqrt{N}} \sum_{k} \xi_{k}(\mathbf{x}) \alpha_{k}\right) \\
& \equiv A\left(\xi(\mathbf{x})+\frac{1}{\sqrt{N}} \chi(\mathbf{x})\right) .
\end{aligned}
$$

Here the operators $\left\{a_{0}, a_{k}\right\}$ are independent creation and destruction operators satisfying the usual creation and destruction operator commutation relations, and the set of functions $\left\{\xi_{0}(\mathbf{x}), \xi_{k}(\mathbf{x})\right\}$ is a complete orthonormal set. This is necessary to produce the mandatory field operator commutation relation $\left[\psi(\mathbf{x}), \psi^{\dagger}\left(\mathbf{x}^{\prime}\right)\right]=\delta\left(\mathbf{x}-\mathbf{x}^{\prime}\right)$.

The meaning of $A$ and $\alpha_{k}$ is essentially as previously defined except that the exact nature of the states involved is not yet defined. The basic issue is that the occupation of the mode described by $a_{0}$ is very large compared to the occupation of any of the modes described by the $a_{k}$. Under these conditions, apart from the notation change $\mathbf{k}$ (momentum) $\rightarrow k$ (an arbitrary label) the relationship between the operators $A, a_{0}, \alpha_{k}, a_{k}$ is exactly the same as in Sect. II.

Notice that $\chi(\mathbf{x})$ has a non-local commutation relation

$$
\begin{aligned}
{\left[\chi(\mathbf{x}), \chi^{\dagger}\left(\mathbf{x}^{\prime}\right)\right] } & =\sum_{k} \xi_{k}(\mathbf{x}) \xi_{k}^{*}\left(\mathbf{x}^{\prime}\right) \\
& =\delta\left(\mathbf{x}-\mathbf{x}^{\prime}\right)-\xi(\mathbf{x}) \xi^{*}\left(\mathbf{x}^{\prime}\right) \\
& \equiv R\left(\mathbf{x}, \mathbf{x}^{\prime}\right)
\end{aligned}
$$

which arises because the $\chi$ operators act only in the subspace orthogonal to the condensate wavefunction $\xi$. These are of the same form as those for similar operators considered by Fetter [7].

\section{Expansion of the Hamiltonian}

The Bogoliubov method will be valid for large $N$ and small $u$, and, as noted above, this is expressed more precisely by setting $u \equiv \tilde{u} / N$, and expanding the Hamiltonian in decreasing powers of $N$ for fixed $\tilde{u}$, after substituting for the field operators using (34). In order to eliminate $a_{0}^{\dagger} a_{0}$ as in the homogeneous case, we note that

$$
\sum_{k} \alpha_{k}^{\dagger} \alpha_{k}=\int d^{3} \mathbf{x} \chi^{\dagger}(\mathbf{x}) \chi(\mathbf{x})
$$

Carrying out this procedure, we then get

$$
H=N \mathcal{H}_{1}+\sqrt{N} \mathcal{H}_{2}+\mathcal{H}_{3}+\ldots
$$

in which

$$
\begin{aligned}
\mathcal{H}_{1}= & -\frac{\hbar^{2}}{2 m} \int d^{3} \mathbf{x} \xi^{*}(\mathbf{x}) \nabla^{2} \xi(\mathbf{x})+\int d^{3} \mathbf{x} \xi^{*}(\mathbf{x}) V(\mathbf{x}) \xi(\mathbf{x}) \\
& +\frac{\tilde{u}}{2} \int d^{3} \mathbf{x}|\xi(\mathbf{x})|^{4} \\
\mathcal{H}_{2}= & -\frac{\hbar^{2}}{2 m} \int d^{3} \mathbf{x}\left\{\chi^{\dagger}(\mathbf{x}) \nabla^{2} \xi(\mathbf{x})+\xi^{*}(\mathbf{x}) \nabla^{2} \chi(\mathbf{x})\right\} \\
& +\int d^{3} \mathbf{x} V(\mathbf{x})\left\{\chi^{\dagger}(\mathbf{x}) \xi(\mathbf{x})+\xi^{*}(\mathbf{x}) \chi(\mathbf{x})\right\} \\
& +\tilde{u} \int d^{3} \mathbf{x}\left\{|\xi(\mathbf{x})|^{2} \xi(\mathbf{x}) \chi^{\dagger}(\mathbf{x})+|\xi(\mathbf{x})|^{2} \xi^{*}(\mathbf{x}) \chi(\mathbf{x})\right\} \\
\mathcal{H}_{3}= & -\frac{\hbar^{2}}{2 m} \int d^{3} \mathbf{x} \chi^{\dagger}(\mathbf{x}) \nabla^{2} \chi(\mathbf{x})+\int d^{3} \mathbf{x} \chi^{\dagger}(\mathbf{x}) V(\mathbf{x}) \chi(\mathbf{x}) \\
+ & \int d^{3} \mathbf{x}\left\{\frac{\tilde{u}}{2}\left(\xi(\mathbf{x}) \chi^{\dagger}(\mathbf{x})\right)^{2}+\frac{\tilde{u}}{2}\left(\xi^{*}(\mathbf{x}) \chi(\mathbf{x})\right)^{2}\right. \\
+ & \left.\chi^{\dagger}(\mathbf{x}) \chi(\mathbf{x})\left(2 \tilde{u}|\xi(\mathbf{x})|^{2}-\mu\right)\right\}-\frac{\tilde{u}}{2} \int d^{3} \mathbf{y}|\xi(\mathbf{y})|^{4}
\end{aligned}
$$

in which

$$
\begin{array}{r}
\mu=\int d^{3} \mathbf{y}\left(\xi^{*}(\mathbf{y})\left(-\frac{\hbar^{2} \nabla^{2}}{2 m}\right) \xi(\mathbf{y})\right. \\
\left.+V(\mathbf{y})|\xi(\mathbf{y})|^{2}+\tilde{u}|\xi(\mathbf{y})|^{4}\right)
\end{array}
$$

If we now choose $\xi$ to minimize $\mathcal{H}_{1}$, subject to the condition that $\int d^{3} \mathbf{x}|\xi(\mathbf{x})|^{2}=1$, we find that

1. The condition for a local minimum of $\mathcal{H}_{1}$ is that the condensate wavefunction $\xi(\mathbf{x})$ satisfies the time independent Gross-Pitaevskii equation 


$$
-\frac{\hbar^{2}}{2 m} \nabla^{2} \xi(\mathbf{x})+V(\mathbf{x}) \xi(\mathbf{x})+\tilde{u}|\xi(\mathbf{x})|^{2} \xi(\mathbf{x})=\mu \xi(\mathbf{x}) .
$$

Here $\mu$ arises as a Lagrange multiplier necessary to maintain the normalization of $\xi(\mathbf{x})$ - any valid value of $\mu$ is permitted in this procedure, and the value obtained is consistent with (44)

2. Under this condition the terms linear in $\chi$ or $\chi^{\dagger}$ vanish, since the $\xi_{k}(\mathbf{x})$ are a set of functions orthogonal to $\xi(\mathbf{x})$.

Since $N$ is known, (45) is to be considered as a nonlinear eigenvalue equation for $\xi(\mathbf{x})$. Thus, the possible values of $\mu$ are the eigenvalues of the Gross-Pitaevskii equation.

For stability $\mathcal{H}_{3}$ should be positive definite; whether this can be satisfied seems to depend on the actual solution of the Gross-Pitaevskii equation which is under consideration. If the condition is not satisfied, the state represented by the solution of the Gross-Pitaevskii equation is not stable, and the extremum found is not a minimum.

When restricted to a fixed $N$ subspace, the expansion (40,41, 43), is exactly the same as that obtained by Fetter (7) using the method based on the minimization of $\langle K\rangle$.

\section{Diagonalization of $\mathcal{H}_{3}$}

The expression (43) for $\mathcal{H}_{3}$ can be diagonalized most simply by working in the $\xi_{k}$ basis, in much the same way as Javanainen [12]. This method is also preferred here since it generalizes rather straightforwardly to the timedependent situation. Thus one can write

$$
\begin{aligned}
\mathcal{H}_{3}=E_{3} & +\sum_{k, q}\left\{\left(L_{k q}+F_{k q}\right) \alpha_{k}^{\dagger} \alpha_{q}\right. \\
& \left.+\frac{1}{2} G_{k q} \alpha_{k}^{\dagger} \alpha_{q}^{\dagger}+\frac{1}{2} G_{k q}^{*} \alpha_{k} \alpha_{q}\right\}
\end{aligned}
$$

where

$$
\begin{aligned}
L_{k q} & =\int d^{3} \mathbf{x} \xi_{k}^{*}(\mathbf{x})\left\{-\frac{\hbar^{2}}{2 m} \nabla^{2}+V(\mathbf{x})\right\} \xi_{q}(\mathbf{x}) \\
F_{k q} & =\tilde{u} \int d^{3} \mathbf{x} \xi_{k}^{*}(\mathbf{x})\left\{2|\xi(\mathbf{x})|^{2}-\mu\right\} \xi_{q}(\mathbf{x}) \\
G_{k q} & =\tilde{u} \int d^{3} \mathbf{x} \xi_{k}^{*}(\mathbf{x})\left\{\xi(\mathbf{x})^{2}\right\} \xi_{q}(\mathbf{x}) \\
E_{3} & =-\frac{\tilde{u}}{2} \int d^{3} \mathbf{y}|\xi(\mathbf{y})|^{4}
\end{aligned}
$$

\section{Quasiparticles}

The Hamiltonian $\mathcal{H}_{3}$ can be diagonalized by a Bogoliubov transformation of the form

$$
\alpha_{k}=\sum_{m} c_{k m} b_{m}+\sum_{m} s_{k m} b_{m}^{\dagger}
$$

and here $b_{m}$ is a quasiparticle destruction operator. We can then write

$$
\chi(\mathbf{x})=\sum_{m}\left(p_{m}(\mathbf{x}) b_{m}+q_{m}(\mathbf{x}) b_{m}^{\dagger}\right)
$$

with

$$
\begin{aligned}
& p_{m}(\mathbf{x})=\sum_{k} c_{k m} \xi_{k}(\mathbf{x}) \\
& q_{m}(\mathbf{x})=\sum_{k} s_{k m} \xi_{k}(\mathbf{x}) .
\end{aligned}
$$

The diagonalized Hamiltonian is then written

$$
\mathcal{H}_{3}=\hbar \omega_{g}(N)+\sum_{m} \hbar \epsilon_{m}(N) b_{m}^{\dagger} b_{m}
$$

Notice that, even though the $N$ dependence is not always explicitly written, almost everything in the above is a function of $N$.

\section{B. Relationship between ground states for $N$ and $N+1$ particles}

The operator $A$ contains an $N$ dependence which arises from the change in the shape of the ground state wavefunction $\xi(\mathbf{x})$ as $N$ changes. This is an effect which does not arise in a spatially homogeneous situation. Thus we can exhibit this feature by writing, as a result of (33),

$$
a_{0}(N)=\int d^{3} \mathbf{x} \xi^{*}(\mathbf{x}, N) \psi(\mathbf{x}),
$$

where the explicit dependence on $N$ of both $\xi$ and $a_{0}$ has now been written.

This means that $a_{0}^{\dagger}(N)|N, \mathbf{n}=\mathbf{0}\rangle$ is a state with $N+1$ particles in the wavefunction corresponding to ground state of the $N$ particle state; it is not the ground state for $N+1$ particles. In other words, $a_{0}^{\dagger}(N)$ is not the operator which converts an $N$ particle ground state into an $N+1$ particle ground state.

We will therefore compute the appropriate operator. We can write

$$
\begin{aligned}
a_{0}(N+1) & =a_{0}(N) \\
& +\int d^{3} \mathbf{x}\left(\xi^{*}(\mathbf{x}, N+1)-\xi^{*}(\mathbf{x}, N)\right) \psi(\mathbf{x}) \\
& \approx a_{0}(N)+\int d^{3} \mathbf{x} \frac{\partial \xi^{*}(\mathbf{x}, N)}{\partial N} \psi(\mathbf{x}) .
\end{aligned}
$$


Now expand $\partial \xi^{*}(\mathbf{x}, N) / \partial N$ in the $N$ particle basis states as

$$
\frac{\partial \xi^{*}(\mathbf{x}, N)}{\partial N}=\frac{1}{N}\left(i r_{0} \xi^{*}(\mathbf{x}, N)+\sum_{k} r_{k} \xi_{k}^{*}(\mathbf{x}, N)\right)
$$

The requirement that $\xi$ be normalized allows us to deduce that $r_{0}$ is real, and by redefining $\xi(N) \rightarrow$ $\xi(N) \exp \left\{-i \int d N r_{0}(N) / N\right\}$ we can transform $r_{0}$ to zero; that is, we can choose a family of solutions of the time-independent Gross-Pitaevskii equation in which the relative phases of the members of the family are such that $r_{0}=0$. Once $r_{0}$ has been eliminated, it is clear that $r_{k}$ are of order of magnitude 1 . This means that we can write approximately

$$
a_{0}(N+1) \approx a_{0}(N)+\frac{1}{N} \sum_{k} r_{k} a_{k}(N)
$$

We now want to relate the $N$ particle ground state to the $N+1$ particle ground state. We can write

$$
\begin{aligned}
|N\rangle_{N} & \equiv \frac{\left\{a_{0}^{\dagger}(N)\right\}^{N}}{\sqrt{N !}}|0\rangle \\
|N+1\rangle_{N+1} & \equiv \frac{\left\{a_{0}^{\dagger}(N+1)\right\}^{N+1}}{\sqrt{(N+1) !}}|0\rangle .
\end{aligned}
$$

We first write (60) in terms of the phonon operators $\alpha_{k}(N)$ thus

$$
a_{0}(N+1)=a_{0}(N)\left(1+\sum_{k} \frac{r_{k}}{N} \frac{\alpha_{k}(N)}{\sqrt{N}}\right) .
$$

and raise this to the power $N+1$, using the binomial theorem to get, accurate to order $1 / N$,

$$
\left\{a_{0}^{\dagger}(N+1)\right\}^{N+1} \approx\left(1+\sum_{k} \frac{r_{k}^{*} \alpha_{k}^{\dagger}(N)}{\sqrt{N}}\right)\left\{a_{0}^{\dagger}(N)\right\}^{N+1} .
$$

From $(61,62$ ) this means that

$$
\begin{aligned}
|N+1\rangle_{N+1} & =\frac{\left\{a_{0}^{\dagger}(N+1)\right\}^{N+1}}{\sqrt{(N+1) !}} \frac{\left\{a_{0}(N)\right\}^{N}}{\sqrt{N !}}|0\rangle \\
& \approx\left(1+\frac{\sum_{k} r_{k}^{*} \alpha_{k}^{\dagger}}{\sqrt{N}}\right) \frac{a_{0}^{\dagger}(N)}{\sqrt{N+1}}|N\rangle_{N}
\end{aligned}
$$

Similar procedures can be carried out for the $a_{k}(N)$ and hence the $\alpha_{k}(N)$, but the differences between these at $N$ and $N+1$ will have a negligible effect because of their small occupation numbers.

From the above, we can say that the operator $B^{\dagger}(N)$ which connects the $N$ and $N+1$ ground states through

$$
B^{\dagger}(N)|N\rangle_{N}=\sqrt{N+1}|N+1\rangle_{N+1}
$$

is given approximately by (to order $1 / N$ )

$$
B^{\dagger}(N) \approx\left(1+\frac{1}{\sqrt{N}} \sum_{k} r_{k}^{*} \alpha_{k}^{\dagger}\right) a_{0}^{\dagger}(N)
$$

This means that the field operator expansion (35) now takes the form

$$
\psi(\mathbf{x}) \approx B(N)\left(\xi(\mathbf{x})+\frac{1}{\sqrt{N}} \chi_{B}(\mathbf{x})\right),
$$

where

$$
\begin{aligned}
\chi_{B}(\mathbf{x}) & \equiv \chi(\mathbf{x})-\xi(\mathbf{x}) \sum_{k} r_{k} \alpha_{k} \\
& =\sum_{m}\left(f_{m}(\mathbf{x}) b_{m}+g_{m}(\mathbf{x}) b_{m}^{\dagger}\right)
\end{aligned}
$$

and

$$
\begin{aligned}
& f_{m}(\mathbf{x})=p_{m}(\mathbf{x})-\xi(\mathbf{x}) \sum_{m} r_{k} c_{k m} \\
& g_{m}(\mathbf{x})=q_{m}(\mathbf{x})-\xi(\mathbf{x}) \sum_{m} r_{k} s_{k m}
\end{aligned}
$$

The physical distinction between the quasiparticle wavefunctions $p_{m}, q_{m}$ and the modified wavefunctions $f_{m}, g_{m}$ is that the first set are amplitudes for the production or absorption of quasiparticle without changing $N, e . g$. , by application of sound waves, whereas the second set give the corresponding amplitudes for changing quasiparticle numbers by changing the particle number by 1 .

It is particularly interesting to see that the corrections to the quasiparticle term are of the same order of magnitude as the original terms; the correction is thus very significant. It reflects the fact that the change in the groundstate wavefunction from $N$ to $N+1$ particles affects the $N$ particles already present as well as the added particle.

\section{TIME-DEPENDENT SOLUTIONS}

Suppose we now consider a representation like (33 35) in which however we have a time-dependent condensate wavefunction $\xi(\mathbf{x}, t)$. This is with fixed $N$ since we are not considering condensate growth; rather, we are investigating the situation where the condensate has been macroscopically disturbed from the stationary state as in the recent experiments in JILA [29] and MIT [30].

Let us proceed within the Schrödinger picture as follows. Firstly suppose the condensate wavefunction (normalized to 1$)$ is $\xi(\mathbf{x}, t)$ and define condensate and noncondensate parts of the field operator by 


$$
\begin{aligned}
\psi_{0}(\mathbf{x}, t) & =\xi(\mathbf{x}, t) \int d^{3} \mathbf{x}^{\prime} \xi^{*}\left(\mathbf{x}^{\prime}, t\right) \psi\left(\mathbf{x}^{\prime}\right) \\
& \equiv a_{0}(t) \xi(\mathbf{x}, t) \\
\psi_{\mathrm{nc}}(\mathbf{x}, t) & =\psi(\mathbf{x})-\psi_{0}(\mathbf{x}, t) .
\end{aligned}
$$

We can set up non-condensate mode operators by defining

$$
a_{k}(t)=\int d^{3} \mathbf{x} \xi_{k}^{*}(\mathbf{x}, t) \psi(\mathbf{x})
$$

where $\left\{\xi_{k}(\mathbf{x}, t)\right\}$ are any orthonormal set of wavefunctions orthogonal to the condensate wavefunction $\xi(\mathbf{x}, t)$.

The total number operator is

$$
\mathcal{N}=\int d^{3} \mathbf{x} \psi^{\dagger}(\mathbf{x}) \psi(\mathbf{x})
$$

and is time independent. We can correspondingly define condensate and non-condensate number operators by

$$
\begin{aligned}
\mathcal{N}_{0}(t) & =a_{\mathrm{nc}}^{\dagger}(t) a_{\mathrm{nc}}(t) \\
\mathcal{N}_{\mathrm{nc}}(t) & =\mathcal{N}-\mathcal{N}_{0}(t) \\
& =\int d^{3} \mathbf{x} \psi_{\mathrm{nc}}^{\dagger}(\mathbf{x}, t) \psi_{\mathrm{nc}}(\mathbf{x}, t)
\end{aligned}
$$

The time-dependence of these operators arises because their definitions, which involve the time-dependent wavefunctions $\xi(\mathbf{x}, t), \xi_{k}(\mathbf{x}, t)$, change with time; they are however still operators in the Schrödinger picture.

Using these definitions it is possible to define the operator $A$ essentially as before so that,

$$
a_{0}(t)=\sqrt{1-\frac{N_{\mathrm{nc}}(t)}{N}} A(t) .
$$

Although $A(t)$ is time-dependent, $\mathcal{N}=A^{\dagger}(t) A(t)$ is time independent. The time-dependence of $A(t)$ comes about because of the changing definition of the modes unaffected by its action. That is, $A$ reduces the total number of particles while leaving the number of non-condensate particles the same, but the definitions of the condensate and non-condensate modes are themselves time dependent. Further, although the operators for condensate and non-condensate modes commute, the $A(t)$ operator does not commute exactly with any of $a_{0}(t), a_{0}^{\dagger}(t), a_{k}(t)$ or $a_{k}^{\dagger}(t)$.

It will be particularly important to keep track of all time-dependences which are a result of the the expression of the time-independent Schrödinger picture field operators $\psi(\mathbf{x})$ as projections on the time-dependent basis vectors $\left\{\xi(\mathbf{x}, t), \xi_{k}(\mathbf{x}, t)\right\}$ - the only operator in this basis that does not develop such a time-dependence is the operator $\mathcal{N}$. We will show that a unitary transformation can be introduced which transforms us to a kind of interaction picture, which we shall call the condensate picture, in which we have a description in terms of time-independent phonon creation and destruction operators $\beta_{k}^{\dagger}, \beta_{k}$ corresponding to the time-dependent modes $\xi_{k}(\mathbf{x}, t)$ provided that the condensate wavefunction $\xi(\mathbf{x}, t)$ satisfies the time-dependent Gross-Pitaevskii equation

$$
i \hbar \frac{\partial \xi}{\partial t}=-\frac{\hbar^{2}}{2 m} \nabla^{2} \xi+V \xi+\tilde{u}|\xi|^{2} \xi+\bar{\mu}(t) \xi
$$

where $\bar{\mu}(t)$ is arbitrary, and of course can be eliminated by multiplying $\xi$ by an appropriate time-dependent phase.

\section{A. Time dependence of the phonon operators}

\section{Explicit time-dependence}

Since $\left\{\xi(\mathbf{x}, t), \xi_{k}(\mathbf{x}, t)\right\}$ are a complete orthonormal set, we can define a projector onto the non-condensate modes by

$$
\begin{aligned}
R\left(\mathbf{x}, \mathbf{x}^{\prime}, t\right) & =\sum_{k} \xi_{k}(\mathbf{x}, t) \xi_{k}^{*}\left(\mathbf{x}^{\prime}, t\right) \\
& =\delta\left(\mathbf{x}-\mathbf{x}^{\prime}\right)-\xi(\mathbf{x}, t) \xi^{*}\left(\mathbf{x}^{\prime}, t\right)
\end{aligned}
$$

so that, using a time-dependent version of (33 35),

$$
A(t) \frac{1}{\sqrt{N}} \chi(\mathbf{x}, t) \approx \int d^{3} \mathbf{x}^{\prime} R\left(\mathbf{x}, \mathbf{x}^{\prime}, t\right) \psi\left(\mathbf{x}^{\prime}\right)
$$

and this means that to the lowest order in $\sqrt{N}$ we can write

$$
\chi(\mathbf{x}, t)=\frac{1}{\sqrt{N}} A^{\dagger}(t) \int d^{3} \mathbf{x}^{\prime} R\left(\mathbf{x}, \mathbf{x}^{\prime}, t\right) \psi\left(\mathbf{x}^{\prime}\right)
$$

The explicit time-dependence of $\chi$ arises from the projector and the operator $A^{\dagger}(t)$. We use expression

$$
A^{\dagger}(t) \approx a_{0}^{\dagger}(t)=\int d^{3} \mathbf{x} \xi(\mathbf{x}, t) \psi^{\dagger}(\mathbf{x})
$$

so that

$$
\begin{aligned}
\frac{\partial A^{\dagger}(t)}{\partial t} & \approx \int d^{3} \mathbf{x} \dot{\xi}(\mathbf{x}, t) \psi^{\dagger}(\mathbf{x}) \\
& \approx A^{\dagger}(t) \int d^{3} \mathbf{x} \dot{\xi}(\mathbf{x}, t) \xi^{*}(\mathbf{x}, t)
\end{aligned}
$$

Now using the explicit form (85) for the projector $R$, and resubstituting for $\psi$ in terms of $A$ and $\chi$ we deduce

$$
\begin{aligned}
\frac{\partial \chi(\mathbf{x}, t)}{\partial t} & =\left\{\int d^{3} \mathbf{x}^{\prime} \dot{\xi}\left(\mathbf{x}^{\prime}, t\right) \xi^{*}\left(\mathbf{x}^{\prime}, t\right)\right\} \chi(\mathbf{x}, t) \\
& +\int d^{3} \mathbf{x}^{\prime} \dot{R}\left(\mathbf{x}, \mathbf{x}^{\prime}, t\right)\left\{\sqrt{N} \xi\left(\mathbf{x}^{\prime}, t\right)+\chi\left(\mathbf{x}^{\prime}, t\right)\right\}
\end{aligned}
$$

This time-dependence is only the explicit timedependence, and does not include that arising from the commutator with the Hamiltonian (40). 
2. Expansion of the Hamiltonian and the condensate picture

The Hamiltonian can now be expansied in decreasing powers of $\sqrt{N}$ in exactly the same way as for the time independent case, with appropriate substitutions. However, the largest terms arising from (42) and (91) are both proportional to $\sqrt{N}$, and these must be arranged to cancel, since otherwise $\chi / \sqrt{N}$ would develop a c-number component comparable with $\xi$, invalidating the expansion (35). We will show that this cancellation is only possible if $\xi(\mathbf{x}, t)$ satisfies the time-dependent GrossPitaevskii equation.

The coefficient of $\sqrt{N}$ in 91 can be written as

$$
\int d^{3} \mathbf{x}^{\prime} \dot{R}\left(\mathbf{x}, \mathbf{x}^{\prime}, t\right) \xi\left(\mathbf{x}^{\prime}, t\right)=\frac{i}{\hbar}\left[\mathcal{H}_{R}(t), \chi(\mathbf{x}, t)\right]
$$

in which

$$
\mathcal{H}_{R}(t)=i \hbar \int d^{3} \mathbf{x}\left\{\dot{\xi}(\mathbf{x}, t) \chi^{\dagger}(\mathbf{x}, t)-\dot{\xi}^{*}(\mathbf{x}, t) \chi(\mathbf{x}, t)\right\} .
$$

Here we have used the identity

$$
0=\int d^{3} \mathbf{x}^{\prime} \dot{R}\left(\mathbf{x}, \mathbf{x}^{\prime}, t\right) \xi\left(\mathbf{x}^{\prime}, t\right)+\int d^{3} \mathbf{x}^{\prime} R\left(\mathbf{x}, \mathbf{x}^{\prime}, t\right) \dot{\xi}\left(\mathbf{x}^{\prime}, t\right)
$$

which arises from the fact that $\int d^{3} \mathbf{x}^{\prime} R\left(\mathbf{x}, \mathbf{x}^{\prime}, t\right) \xi\left(\mathbf{x}^{\prime}, t\right)=$ 0$.

If $\xi$ satisfies the time-dependent Gross-Pitaevskii equation (33), it is clear that $\mathcal{H}_{R}=-\mathcal{H}_{2}$. This means that we can make a unitary transformation from the Schrödinger picture to a picture which we call the condensate picture, defined by

$$
\begin{aligned}
|\Phi, t\rangle & \rightarrow V(t)|\Phi, t\rangle \equiv|\Phi, t\rangle_{c} \\
\psi(\mathbf{x}) & \rightarrow V(t) \psi(\mathbf{x}) V^{-1}(t) \equiv \psi_{c}(\mathbf{x}, t) \\
\frac{d}{d t} V(t) & =-\frac{i}{\hbar} \sqrt{N} \mathcal{H}_{R}(t) V(t) .
\end{aligned}
$$

In this picture the part of the explicit time-dependence of $\chi$ proportional to $\sqrt{N}$ in (91) will disappear, and the term $\sqrt{N} H_{2}$ in the equation of motion for the states $|\Phi, t\rangle_{c}$ will cancel with a term arising from the unitary transformation. We will then be able to write the equation of motion in this picture as (to order $N^{0}$ )

$$
\left(N \mathcal{H}_{1}+\mathcal{H}_{3}(t)\right)|\Phi, t\rangle_{c}=i \hbar \frac{d}{d t}|\Phi, t\rangle_{c}
$$

in which

$$
\begin{aligned}
\mathcal{H}_{1} & =-\frac{\hbar^{2}}{2 m} \int d^{3} \mathbf{x} \xi^{*}(\mathbf{x}, t) \nabla^{2} \xi(\mathbf{x}, t) \\
+ & \int d^{3} \mathbf{x} \xi^{*}(\mathbf{x}, t) V(\mathbf{x}) \xi(\mathbf{x}, t)+\frac{\tilde{u}}{2} \int d^{3} \mathbf{x}|\xi(\mathbf{x}, t)|^{4},
\end{aligned}
$$

$$
\begin{aligned}
& \mathcal{H}_{3}(t)=\int d^{3} \mathbf{x}\left[-\frac{\hbar^{2}}{2 m} \chi_{c}^{\dagger}(\mathbf{x}, t) \nabla^{2} \chi_{c}(\mathbf{x}, t)\right. \\
& +\chi_{c}^{\dagger}(\mathbf{x}, t) V(\mathbf{x}) \chi_{c}(\mathbf{x}, t) \\
& +\frac{\tilde{u}}{2}\left(\xi(\mathbf{x}, t) \chi_{c}^{\dagger}(\mathbf{x}, t)\right)^{2}+\frac{\tilde{u}}{2}\left(\xi^{*}(\mathbf{x}, t) \chi_{c}(\mathbf{x}, t)\right)^{2} \\
& \left.+\chi_{c}^{\dagger}(\mathbf{x}, t) \chi_{c}(\mathbf{x}, t)\left[2 \tilde{u}|\xi(\mathbf{x}, t)|^{2}-\mu(t)\right]\right] \\
& \quad-\frac{\tilde{u}}{2} \int d^{3} \mathbf{y}|\xi(\mathbf{y}, t)|^{4} .
\end{aligned}
$$

in which $\mu(t)$ has no connection with the $\bar{\mu}(t)$ in $(83)$, but is given by

$$
\begin{aligned}
\mu(t)= & \int d^{3} \mathbf{y}\left(\xi^{*}(\mathbf{y}, t)\left(-\frac{\hbar^{2} \nabla^{2}}{2 m}\right) \xi(\mathbf{y}, t)\right. \\
& \left.+V(\mathbf{y})|\xi(\mathbf{y}, t)|^{2}+\tilde{u}|\xi(\mathbf{y}, t)|^{4}\right) \\
= & -i \hbar \int d^{3} \mathbf{y} \xi^{*}(\mathbf{y}, t) \frac{\partial \xi(\mathbf{y}, t)}{\partial t}
\end{aligned}
$$

Notice that no time-dependence is written for $\mathcal{H}_{1}$, since it is in fact a constant c-number when $\xi$ satisfies the timedependent Gross-Pitaevskii equation.

Note that although the equation of motion is given by (98), the energy is given by the full Hamiltonian $H=N \mathcal{H}_{1}+\sqrt{N} \mathcal{H}_{2}+\mathcal{H}_{3}(t)$, with the operators evaluated in the condensate picture. Since there is no timedependence of the Schrödinger picture Hamiltonian, the total energy must be conserved.

\section{Time-independent phonon operators}

The expansion of the phonon field in the condensate picture as

$$
\chi_{c}(\mathbf{x}, t)=\sum_{k} \xi_{k}(\mathbf{x}, t) \alpha_{k}
$$

does not automatically require that $\alpha_{k}$ be time independent. However the mode functions $\xi_{k}$ have been so far essentially arbitrary. The explicit time-dependence of $\chi_{c}$ in the condensate picture is given by omitting the term proportional to $\sqrt{N}$ in (91), and by inserting the expansion (103) into this, we find that $\alpha_{k}$ can be chosen to be independent of time if the mode functions satisfy the equation of motion

$$
\begin{aligned}
\frac{\partial \xi_{k}(\mathbf{x}, t)}{\partial t}= & \xi_{k}(\mathbf{x}, t)\left\{\int d^{3} \mathbf{y} \xi^{*}(\mathbf{y}, t) \dot{\xi}(\mathbf{y}, t)\right\} \\
& -\xi(\mathbf{x}, t)\left\{\int d^{3} \mathbf{y} \dot{\xi}^{*}(\mathbf{y}, t) \xi_{k}(\mathbf{y}, t)\right\}
\end{aligned}
$$

It is straightforward to check that the requirements that $\xi_{k}$ form an orthonormal set, and are orthogonal to $\xi$ are both preserved by this equation of motion. 
Substituting the expansion (103) into $\mathcal{H}_{3}(t)$ as given by (100) we obtain $\mathcal{H}_{3}(t)$ in the form

$$
\begin{aligned}
\mathcal{H}_{3}(t)= & E_{3}(t)+\sum_{k, q}\left\{\left(L_{k q}+F_{k q}(t)\right) \alpha_{k}^{\dagger} \alpha_{q}\right. \\
& \left.+G_{k q}(t) \alpha_{k}^{\dagger} \alpha_{q}^{\dagger}+G_{k q}^{*}(t) \alpha_{k} \alpha_{q}\right\} .
\end{aligned}
$$

Here $L_{k q}(t), F_{k q}(t), G_{k q}(t)$ and $E_{3}(t)$ are defined in the same way as in (47 50), but using in this case the condensate picture operators and the time-dependent mode functions which are now arbitrary only at the initial time, since the equation of motion (104) gives them for all future times.

\section{B. Summary of the time-dependent description}

We have shown that the field operators can be written in a time-dependent version of the form (35). This expansion is valid as an asymptotic expansion in $1 / \sqrt{N}$ if and only if $\xi(\mathbf{x}, t)$ satisfied the time dependent GrossPitaevskii equation.

\section{CONCLUSION}

The adapted Bogoliubov method presented in this paper gives a precise meaning to the definition of the macroscopic wavefunction usually defined by the limiting procedure [3]

$$
\left\langle N, m, t\left|\psi^{\dagger}(\mathbf{x})\right| N-1, m, t\right\rangle \sim \sqrt{N} \xi^{*}(\mathbf{x}, t),
$$

where, in the words of [3],

"...since the condensate contains a macroscopically large number of particles, changing this number by 1 does not essentially affect the state of the system; we may say that the result of adding (or removing) one particle in the condensate is to convert a state of the system of $N$ particles into the 'same' state of a system of $N+1$ particles. ... the symbols $|N, m, t\rangle$ and $|N+1, m, t\rangle$ denote two 'like' states differing only as regards the number of particles in the system."

This definition has the disadvantage of being rather vague about exactly what is meant by the concept of the 'same' or 'like' states which differ only in that the values of $N$ differ by 1 . From this paper it is clear that the description of the eigenfunctions of the non-condensed particles depends on the value of $N$, and although the difference between the eigenfunctions for $N$ and $N+1$ must become negligible for $N \rightarrow \infty$, if we are contemplating a situation in which the condensate is growing, as in recent experiments, this difference may have nonnegligible effects.

Our method can also be seen as the logical completion of the aim of Hugenholtz and Pines [26] to treat the Bose condensed gas by elimination the ground state. Their method was only able to do this at the expense of breaking the exact conservation of particle numbers, whereas our method puts the approximation in the relationship between the operators $A, \alpha_{k}$ and the particle operators, and in their exact commutation relations. However, there is no good reason to why one should not in principle seek a more accurate approximation than has been done here by computing terms of higher order in the inverse $\sqrt{N}$ expansion, and get results comparable with those of Hugenholtz and Pines. For the condensates at present in existence this is not an urgent problem; rather the main problem is to get as simple a description as possible of the eigenfunctions as a function of $N$ as well as the other variables.

Griffin [28] has recently shown how the Hartree-FockBogoliubov method can also be used. This is closely related to any Bogoliubov method, including this one, but it suffers from not being a systematic expansion in any parameter. For example, depending on assumptions made on certain averages one may or may not obtain a gapless spectrum. A systematic method would identify all terms of a given order in an appropriate small parameter, and either use all of them or use none of them. If the Hartree-Fock-Bogoliubov method is developed in inverse powers of $\sqrt{N}$, one will find the same results as presented here to the degree of accuracy presented here.

It is well known that the Bogoliubov method is gapless 27.28 (in the long wavelength limit, the energy levels approach the energy of the ground state; i.e., a phonon of very long wavelength has vanishingly small energy). The method of derivation used here shows that the nonconservation of particle numbers, often seen as the hallmark of the method, is not at all essential to the method; that it is really takes only a slightly different point of view to see that the non conservation of particle numbers arises from a slightly inappropriate way of looking at the quasiparticle operators. Nevertheless, the elimination of this problem is absolutely essential if we wish to study condensate growth. Approximations which do not preserve particle number conservation can give rise to spurious terms in the equations of motion for the development of a condensate, and thus make it difficult to identify the true details of the growth process.

The reason for developing the modified Bogoliubov method is thus to apply the method to the growth of the condensate, and this will require its incorporation into the framework of Quantum Kinetic Theory [31]. If the condensate growth is rather slow, it will adequate to use the time-independent formalism of Sect. [II], but for faster condensate growth, the time-dependent formalism will be indispensable. These aspects will be treated in 32. 


\section{ACKNOWLEDGMENTS}

I would like to thank Rob Ballagh and Keith Burnett for helpful comments. This work was supported by the Marsden Fund under contract number PVT-603.

[1] N.N. Bogoliubov, J.Phys. (USSR), 11, 23, (1947); reprinted in D. Pines, The Many Body Problem, Benjamin N.Y. (1962);

[2] A.A. Abrikosov, L.P. Gorkov and I.E. Dzyaloshinski, Methods of Quantum Field Theory in Statistical Physics, Dover NY (1963);

[3] E.M. Lifshitz and L.P. Pitaevskii, Statistical Physics Part 2, Landau and Lifshitz Course of Theoretical Physics Vol. 9 (Pergamon Press, Oxford 1980)

[4] A.M. Fetter, Quantum Theory of Many Body Systems, McGraw Hill (N.Y. 1971)

[5] V.L. Ginzburg and L.P. Pitaevskii, Zh. Eksp. Teor.Fiz. 34, 1240 (1958) [Sov. Phys. JETP 7, 858 (1958)]; E.P. Gross, J. Math. Phys. 4, 195 (1963).

[6] M. Anderson, J.R. Ensher, M.R. Matthews, C.E. Wieman and E.A. Cornell, Science 269, 198 (1995); C.C. Bradley, C.A. Sackett, J.J. Tollet, and R. Hulet, Phys. Rev. Lett. 75, 1687 (1995); K.B. Davis, M-O.Mewes. M.R. Andrews. N.J. van Druten, D.S. Durfee, D.M. Kurn, and W. Ketterle, Phys. Rev. Lett. 75, 3969 (1995); M.-O. Mewes et al. , Phys. Rev. Lett. 77, 416 (1996).

[7] A. L. Fetter, Ann. Phys. (N.Y.) 70, 67, (1972)

[8] A. L. Fetter, Phys. Rev. A 53, 4246 (1996)

[9] M. Lewenstein and L. You, Phys. Rev. A 53, 909 (1996);

[10] L. You, W. Hoston, M. Lewenstein, and K. Huang, preprint.

[11] M. Lewenstein, L. You, Phys. Rev. Lett. 77, 3489, (1996)

[12] J. Javanainen, Phys. Rev. A 5, 3722, (1996)

[13] M. Edwards, R.J. Dodd, C. Clarke and K. Burnett, Journal of Research of the National Institute of Standards of Standards and Technology 101, 553, (1996)

[14] P. Ruprecht, M.J. Holland, K. Burnett, and M. Edwards, Phys. Rev. A 51, 4704 (1995).

[15] M. Edwards and K. Burnett., Phys. Rev. A 51, 1382 (1995)

[16] S.A. Morgan, R. Ballagh and K. Burnett, Phys. Rev. A, to appear

[17] M. Edwards, R. J. Dodd, C. W. Clark, P. A. Ruprecht, K. Burnett, Phys. Rev. A 53, R1950 (1996).

[18] Mark Edwards, P. A. Ruprecht, K. Burnett, R. J. Dodd, and Charles W. Clark, Phys. Rev. Lett., Phys. Rev. Lett. 77, Aug (1996).

[19] G. Baym and C.J. Pethick, Phys. Rev. Lett. 76, 6 (1996).

[20] S. Stringari, Phys. Rev. Lett. 76, 1405 (1996).

[21] F. Dalfovo, and S. Stringari, Phys. Rev. A 53, 2477 (1996).

[22] S. Stringari, Phys. Rev. Lett 76,2360 (1996).

[23] L.You and M. Holland, Phys. Rev. A 53,1 (1996).
[24] M.J. Holland and J. Cooper, Phys. Rev. A, 53, R1954 (1996).

[25] T.D. Lee, K. Huang and C.N. Yang, Phys.Rev. 106, $1135,(1957)$

[26] N.M. Hugenholtz and D. Pines, Phys Rev. 116, 489, (1959)

[27] P.C. Hohenberg, P.C. Martin, Ann. Phys (NY) 34, 291 (1965)

[28] A. Griffin, Phys. Rev. B 53, 9341, (1996)

[29] D.S. Jin, J. R. Ensher, M.R. Matthews, C. E. Wieman, E.A. Cornell, Phys. Rev. Lett. 77, 420 (1996).

[30] M.-O. Mewes et al., Phys. Rev. Lett. (in press).

[31] C. W Gardiner, P. Zoller, Quantum Kinetic Theory I: A quantum kinetic master equation for condensation of a weakly interacting Bose gas without a trapping potential, to appear in Phys. Rev. A

[32] C. W Gardiner, P. Zoller, Quantum Kinetic Theory II, in preparation. 